\title{
一种能量有效的传感器监测网络成簇协议
}

沈 洋 $^{1+}$, 齐望东 ${ }^{1}$, 戴 浩 $^{2}$

${ }^{1}$ (解放军理工大学 计算机工程系,江苏 南京 210007)

${ }^{2}$ (中国电子设备系统工程公司, 北京 100039)

\section{An Energy Efficient Clustering Protocol for Surveillance Sensor Networks}

\author{
SHEN Yang ${ }^{1+}, \quad$ QI Wang-Dong ${ }^{1}, \quad$ DAI Hao ${ }^{2}$ \\ ${ }^{1}$ (Department of Computer Engineering, PLA University of Science and Technology, Nanjing 210007, China) \\ ${ }^{2}$ (Chinese Electronic Equipment and System Engineering Corporation, Beijing 100039, China) \\ + Corresponding author: E-mail: sy197610@163.com
}

Shen Y, Qi WD, Dai H. An energy efficient clustering protocol for surveillance sensor networks. Journal of Software, 2008,19(9):2432-2441. http://www.jos.org.cn/1000-9825/19/2432.htm

\begin{abstract}
This paper proposes a distributed energy efficient clustering protocol for target surveillance in sensor networks (EECTS). The protocol selects cluster heads according to a hybrid of node's residual energy and distribution of its neighbors. In addition, for the sake of reducing the energy dissipation of the cluster head, a minimum spanning tree with root of the base station is constructed among the cluster heads. Then it sends the gathered data to its upstream node along the spanning tree. The chief task of surveillance sensor networks is to sensing the moving target. So an intra-cluster node schedule method named EECTS-1 that can senses the most part of the network and it's enhanced method EECTS-2 are introduced. The two methods can obtain the high continuous surveillance degree when the moving target enters into the network. EECTS produces a linear network lifetime in the number of nodes and keeps good continuous surveillance degree simultaneously. The simulation results show that with the same performance of surveillance the EECTS-1 achieves the same lifetime as that HEED obtains and outperforms DEEG with up to $35 \%$ improvement. The EECTS-2 outperforms HEED significantly with prolonging the network lifetime about $70 \% \sim 80 \%$. Therefore the EECTS is suitable for military target surveillance and has high reliability of the sensing information.
\end{abstract}

Key words: surveillance sensor network; cluster; minimum spanning tree; continuous surveillance degree; coverage

摘 要: 提出了一种分布式能量有效的传感器监测网络成簇协议 EECTS(energy-efficient clustering protocol for target surveillance). 在该协议中, 节点根据邻居节点的分布情况以及自己的剩余能量来竞争簇首. 为了降低簇首的能 量消耗,簇首间形成一个以基站为根的最小生成树,将监测到的数据通过多跳方式直接发送到生成树的上游节点.由 于监测网络的首要任务是能够对移动目标进行不间断的监测, 此协议还提出了一种簇内调度方法 EECTS-1, 可监测 到网络中的大部分区域, 并在此基础上提出了改进方法 EECTS-2. 这两种方法在目标进入网络时都能取得较高的持 续监测度. 随着节点密度的提高, EECTS 协议在保证监测性能的前提下, 使得传感器监测网络的寿命相应于节点数 
量呈线性增长.仿真结果表明, 在监测性能相同的情况下, 运行 EECTS-1 协议的网络寿命与 HEED 协议相同, 比 DEEG 协议的网络寿命延长了约 35\%,EECTS-2 协议比 EECTS-1 和 HEED 协议的网络寿命延长了约 $70 \%$ 80\%.EECTS 成簇协议可应用于军事目标监测等领域,工作时间长,监测结果具有很高的可靠性.

关键词: 传感器监测网络;簇;最小生成树;持续监测度;覆盖

中图法分类号: TP393

文献标识码: A

近年来,随着传感器技术、微机电系统(micro-electro-mechanism system)、嵌入式技术以及低功耗无线通信 技术的发展,使得部署具有无线通信和板载信号处理能力的微型低能量传感器成为可能. 这些廉价的、低功耗 的传感器节点共同组织成无线传感器网络, 通过节点间的相互协作, 将其监测到的多种感知信息(如磁场强度、 温度、湿度等)传送到基站进行处理.战场监测及入侵检测 ${ }^{[1-4]}$ 是目前传感器网络的一个重要的应用方向. 在这类 应用中, 由于传感器节点有严格的能量限制, 而且往往部署在遥远或危险的区域, 使得节点难以进行能量的补 充, 这些因素决定了传感器的生存时间是有限的. 为了延长网络的生存时间, 更好地完成监测任务, 需要设计能 量有效的协议以适应传感器监测网络的特点.

分簇是一种比较理想的节点组织形式,许多能量有效的协议都是在簇结构的基础上设计完成的 ${ }^{[5,6]}$.族结构 具有很多优点, 例如, 由簇首节点担负数据融合的任务, 减少了数据通信量 ${ }^{[7,8]}$; 分簇式的拓扑结构有利于分布式 算法的应用,适合大规模部署的网络, 扩展性强; 由于大部分节点在相当长的时间内关闭通信模块, 所以显著地 延长了整个网络的生存时间.

本文提出了一种适合移动目标监测的新的能量有效成簇协议 EECTS(energy-efficient clustering protocol for target surveillance). 该算法让节点轮流成为簇首以均衡能量, 簇首的选举概率与节点剩余能量及周围相邻节 点的分布相关. 簇形成后, 簇首让一部分成员处于监测状态, 其他节点进入睡眠状态, 在取得较高监测性能的基 础上又节约了能量.当节点监测到外来目标后向簇首汇报,簇首通过多跳路由将信息传回基站.

本文第 1 节是无线传感器网络成簇算法的相关研究. 第 2 节是问题描述及系统模型. 第 3 节对 EECTS 算法 进行详细的描述.第 4 节是仿真结果及性能分析.最后对全文进行总结并指出下一步工作的研究方向.

\section{1 相关工作}

$\mathrm{LEACH}^{[9]}$ 是一种分布式自组织协议,通过减少与基站直接通信的节点数量来达到节能的目的.LEACH 协 议按轮 (round)运行, 每轮分为簇建立阶段和簇稳定阶段. 在簇建立阶段, 网络随机选出部分传感器节点作为簇 首,接着,选出的簇首进行广播,其他节点根据接受信号的强弱来选择最近的簇首加入,并与该簇首一起形成簇. 同时, 簇首在簇内进行 TDMA 调度, 为每一个成员分配一个时隙, 节点在其余时隙内处于睡眠状态, 既节约了能 量也避免了节点间的通信冲突. 在簇稳定阶段中, 簇中的成员把数据传送给簇首, 簇首收集完一帧的数据后对数 据进行聚合,然后发往基站.但 LEACH 协议不能保证簇首分布均匀,因而使得簇首分布不均时簇内的通信不能 够满足自由空间模型, 导致能耗增加. 由于采用了 TDMA 调度方式, 当簇较大时, 该簇内会包含较多的的成员, 使 得 TDMA 一帧较长, 而簇成员只在分配的时隙内处于活动状态,网络可能无法持续对入侵目标进行监视, 导致监 测性能下降.传感器监测网络的范围往往比较大,监测的数据通过多跳路由传送回基站,而 LEACH 采用的是簇 首和基站间的单跳通信方式,无法应用到实际环境中.

$\mathrm{HEED}^{[10]}$ 也是一种完全分布式的成簇算法. 节点根据剩余能量和簇内 通信费用两个参数计算出自己成为临时簇首的概率,经过 $O(1)$ 次循环后,临 时簇首可以判断出自己是否成为簇首. HEED 保证了簇首分布是均匀的, 数 据经过多跳路由传回基站.但 HEED 在选举簇首时没有考虑到簇内节点的 分布情况, 可能会造成簇内节点分布不均匀,如图 1 所示. 与 LEACH 协议一 样,HEED 算法采用的也是 TDMA 调度方式,当簇较大时不利于监测任务的 完成.

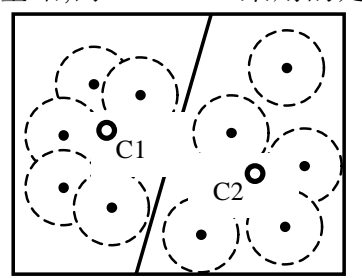

Fig.1 Different cluster structures 图 1 不同簇结构 
$\mathrm{DEEG}^{[11]}$ 与 HEED 类似,节点根据剩余能量和接收信号的强弱经过若干次循环后决定自己是否能够成为簇 首.DEEG 保证了簇首分布均匀, 数据经过多跳路由传送回基站. 但 DEEG 算法是从簇成员中随机选出固定数目 的节点, 使其处于工作状态, 没有考虑到簇内节点的分布, 导致了监测区域的重叠, 增加了不必要的能量消耗, 因 此也不适用于传感器监测网络.

传感器监测网络的首要任务是能够对进入网络的移动目标不间断地进行监测, 上述分簇算法其设计目标 主要是针对周期性的数据传输应用,没有考虑到移动目标监测网络的应用需求.

\section{2 问题描述及系统模型}

EECTS 算法与文献[10-15]中的算法类似,采用的也是按轮方式运行.每轮由簇生成、生成树创建和网络监 测 3 部分组成.

\section{1 网络模型}

$N$ 个节点通过飞机布设或炮弹抛酒在一个区域中,并具有以下特点:

- 节点部署后就不再移动,自组织成网.

- 每个节点知道自己及基站的位置信息.

- 所有的节点具有相同的能力.

- 节点的发射功率可以控制.

第 2 假设中节点的位置可以事先由定位算法 ${ }^{[16,17]}$ 获得. 在文献[10,11]中, 节点根据接收信号的强弱来竞争 簇首, 由于它们的通信模型使用的是类似第 3.2 节中的模型, 接收信号的强弱是从模型中推出来的, 所以从仿真 角度上说与节点知道自己位置的设想是一致的.

\section{2 通信模型}

本文采用与文献[18]中相同的无线通信模型, 见式(1).

$$
\begin{aligned}
& E_{T x}(l, d)=E_{T x-\text { elec }}(l)+E_{T x-a m p}(l, d)=\left\{\begin{array}{l}
l E_{\text {elec }}+l \varepsilon_{f s} d^{2}, d<d_{0} \\
l E_{\text {elec }}+l \varepsilon_{m p} d^{4}, d \geq d_{0}
\end{array}\right. \\
& E_{R x}(l)=E_{R x-\text { elec }}(l)=l E_{\text {elec }}
\end{aligned}
$$

$E_{T x}(l, d)$ 表示将 $l$ 比特长的信息发送到 $d$ 米外所需要的发送能量, $E_{R x}(l)$ 表示接收 $l$ 比特的信息所需的能量.门限值 $d_{0}$ 是一个常数,根据文献[18]中的定义可知, 在采用发射和接收天线的增益均为 1 , 天线高度为 $1.5 \mathrm{~m}$ 及 $914 \mathrm{MHz}$ 的电台频率下, $d_{0} \approx 87 \mathrm{~m}$. 当发送节点与接收节点的距离小于 $d_{0}$ 时, 发送方发送数据的能量损耗与距离的平 方成正比,否则与距离的四次方成正比. $E_{\text {elec }}$ 表示无线收发电路所消耗的能量, $\varepsilon_{f s} d^{2}$ 或 $\varepsilon_{m p} d^{4}$ 表示放大器消耗的 能量,其值由发送节点与接收节点间的距离以及可接受的位错误率决定.

\section{3 问题描述}

对于探测移动目标的传感器监测网络来说, 能够不间断地对目标进行监测是设计成簇算法最关键的因素. 同时由于无线传感器节点的能量有限, 为了最大限度地延长监测网络的生存周期, 在满足一定监测性能的前提 下尽可能地减少能量消耗也是设计成簇算法时要考虑的一个重要因素. 所以簇算法应当满足以下几方面的 条件:

(1) 由于监测网络的范围很广, 要求算法的可扩展性强, 因此簇生成算法应当是分布式的, 每个节点根据本 地信息独立地决定自己的状态.

(2) 簇内节点的分布应当比较均匀, 以利于完成监测任务. 如图 1 所示, 从图中能看出簇 C2 中节点的分布比 簇 C1 中的要均匀,当移动目标进入簇 C2 的区域时可以被网络及时地、不间断地监测到.

(3) 簇首分布应当是均匀的 ${ }^{[11]}$, 使得节点间通信的能量损耗尽量满足自由空间模型,即能量消耗与距离的 平方成正比. 
(4) 簇首应当选择剩余能量较多且其邻节点分布均匀的节点.

\section{EECTS 协议描述}

EECTS 算法采用按轮方式运行,每轮由簇的生成、生成树创建和网络监测 3 部分组成.在簇的生成阶段,在 各个节点明确自己的状态后, 簇首选择一部分工作节点, 这些工作节点可以监测到簇内大部分区域, 而其他成员 节点进入睡眠状态以节约能量. 在生成树创建阶段, 基站首先发送广播,收到该广播的簇首进行转发并选择自己 的下一跳,最后形成一个以基站为根的生成树.由于在传感器监测网络中对监测数据的实时性要求较高,所以生 成树上的父节点一旦接收到子节点的数据后直接向上游节点转发,而不是等待接收其他子节点的数据汇聚后 再转发.在网络监测阶段,苏醒的节点发现到目标后向簇首汇报,监测阶段的时间远大于簇的生成时间与生成树 创建时间之和,从而保证了网络的有效工作时间.

\section{1 簇生成算法}

\subsection{1 簇首选择}

对移动目标进行监视的传感器监测网络最主要的功能是能够对目标不间断地进行监测, 因此在簇形成后, 簇内的节点应当像图 1 中的 C2 簇一样分布得比较均匀,这样,当外来目标进入该簇后,网络可以对目标进行持续 的监视.EECTS 算法中簇的大小都是半径为 $R$ 的区域. 在簇首选择过程中首先设置一个初始簇首比例 $P_{\text {init }}$ 该值 的大小与节点的通信范围和部署区域有关. 由于各个节点的能量消耗速度不一致, 为了防止低能量的节点当选 簇首而造成整个簇的失效,将节点的剩余能量作为选择簇首的基本参数.定义节点当选簇首的概率为

$$
C H_{\text {prob }}=p_{\text {init }} \times \frac{E_{\text {cur }}}{E_{\text {init }}}
$$

其中, $E_{c u r}$ 表示节点当前的能量值, $E_{i n i t}$ 表示节点的初始能量值, 当节点的能量小于门限值 $E_{\min }\left(\right.$ 如 $10^{-4}$ )后认为节 点死亡.节点每过一段时间进行一次 hello 消息的交换,通过交换节点可以获得相邻节点的信息,计算出自己与 邻节点的平均距离 $E$ 及距离均方差 $D . E$ 越小说明该节点和相邻节点间的通信费用越小, $D$ 越大说明邻节点的分 布越均匀. 在簇首选择过程中把 $E$ 和 $D$ 作为辅助参数, 将 $\frac{\beta D}{\alpha E}$ 定义为节点能否当选簇首的优先级, $\alpha, \beta$ 分别代表了 $E$ 和 $D$ 在优先级中所占的权重.

算法. 簇首选择算法.

$S_{\text {neibor }}(i) \leftarrow\{v \mid v$ lies in $i$ range $\}$

calculate $\mathrm{CH}_{\text {prob }}$ and pri of $\mathrm{I}$

is_ch $=$ false

If $C H_{\text {prob }}>\operatorname{Random}(0,1)$ and $E_{\text {cur }}>E_{\text {min }}$

Set myself tentative_clusterhead

Broadcast $(i \rightarrow i d, i \rightarrow$ pri,my_status)

...After $t$

If tentative_set $=$ !empty and my_status=tentative_clusterhead

max_pri=MAX_PRI(all nodes in tentative_set)

If $m y \_p r i>\max \_p r i$

is_ch=true

Broadcast(i->id,i->pri,my_status)

If tentative_set $=$ !empty and my_status=tentative_clusterhead

max_pri=MAX_PRI(all nodes in tentative_set)

If $m y \_p r i>\max \_p r i$

is_ch=true 
Broadcast(i->id,i->pri,my_status)

Else if tentative_set=empty and my_status=tentative_clusterhead

is_ch=true

Broadcast(i->id,i->pri,my_status)

Else

Add new_clusterhead to clusterhead_set

...After $2 t$

If is_ch=false and clusterhead_set!=empty

Join_cluster(clusterhead $k$ which has the max pri in my clusterhead_set)

Else if is_ch=false and clusterhead_set=empty //some node maybe separate from others is_ch=true

在每轮开始时, 节点计算自己当选簇首的概率 $C H_{p r o b}$ 和优先级 $p r i=\frac{\beta D}{\alpha E}$, 如果概率大于某个值, 则声明自己 是临时簇首,将自己的节点标识、优先级和状态(普通节点或临时簇首或族首)广播出去并等待一段时间 $t, t$ 的长 度要保证节点可以收到相邻所有临时族首发送的消息.在等待 $t$ 时间后, 临时簇首检查自己收到的其他临时族 首的优先级,如果自己的优先级最高, 则当选簇首并广播,如果没有收到任何其他的临时簇首信息,则直接当选 簇首并广播.在经过 $2 t$ 时间后每个节点检查自己的状态,如果是普通节点且收到了簇首声明,则将自己加入到优 先级最大的那个簇, 如果没有收到任何簇首声明, 则说明当前的节点是一个孤立节点, 它的邻节点与其距离大于 通信半径 $R$, 节点直接将自己声明为簇首.

\section{1 .2 簇内调度}

在监视移动目标的传感器监测网络中为了延长网络的生存时间, 节点部署往往非常密集, 可以达到 20 个/

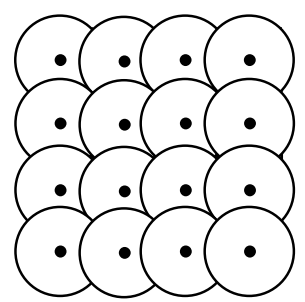

Fig.2 Coverage model 图 2 覆盖模型 米 ${ }^{3[19]}$.这样, 每个簇中的节点数目也相当可观, 按照传统的 TDMA 调度方式会造成 一帧过长,在监测的时间段上产生很多的“监测真空”,降低了监测的精度.EECTS 算 法从簇成员中选择一部分节点处于工作状态, 其他节点休眠.这些工作节点能够监 测到簇内的绝大部分区域,这样既保证了一定的监测精度, 又提高了能量的利用率.

由于簇首知道自己簇内所有成员的位置信息,族内调度采用一种中心化的方 式计算.簇首将簇分成若干个半径为 $r$ 的圆格, $r$ 是节点的探测半径,如图 2 所示. 理 想情况下, 如果节点落在了圆心处, 则此格被完全覆盖. 族首根据成员的位置将其归 属到不同的圆格中,并从每个格个中选择距离圆格中心最近的成员作为工作节点, 格中其他节点进入睡眠状态.

由文献[20]可知,随机分布的节点可以被看作为一个泊松点过程, 它有以下两个性质: 1) 如果节点的分布区 域为 $S$, 泊松点过程的节点密度为 $\lambda$, 在区域 $A$ 中的节点数目 $N(A)$ 等于 $k$ 的概率为 $P(N(A)=k)=\frac{\mathrm{e}^{-\lambda\|A\|}(\lambda\|A\|)^{k}}{k !}$, 其中 $A$ 是 $S$ 的一个子区域, $\|A\|$ 表示 $A$ 的面积; (2) 在区域 $A$ 中给定 $n$ 个点,它们的位置是相互独立的随机变量且 在 $A$ 中均匀分布. $d_{i}$ 是一个随机变量, 表示图 2 圆格中的点 $\left(x_{i}, y_{i}\right)$ 到圆心的距离, 则圆格中所有的点到圆心的期 望为

$$
E\left(d_{i}\right)=\iint_{A} \sqrt{x_{i}^{2}+y_{i}^{2}} \rho\left(x_{i}, y_{i}\right) \mathrm{d} x \mathrm{~d} y=\iint_{A} r \rho(r, \theta) \mathrm{d} r \mathrm{~d} \theta
$$

其中 $A$ 代表了圆格区域,由上述的泊松点过程定义可知, $\rho(r, \theta)=\frac{1}{\|A\|}=\frac{1}{\pi r^{2}}$, 所以,

$$
E\left(d_{i}\right)=\int_{0}^{2 \pi} \int_{0}^{r} r^{2} \rho \mathrm{d} r \mathrm{~d} \theta=2 \pi \rho r^{3} / 3=2 r / 3
$$


同理,

$d_{i}$ 的均方差为

$$
E\left(d_{i}^{2}\right)=\iint_{A}\left(x_{i}^{2}+y_{i}^{2}\right) \rho\left(x_{i}, y_{i}\right) \mathrm{d} x \mathrm{~d} y=r^{2} / 2
$$

$$
D\left(d_{i}\right)=\sqrt{E\left(d_{i}{ }^{2}\right)-E^{2}\left(d_{i}\right)}=r / 3 \sqrt{2}
$$

因此, 圆格中离圆心最近的节点与圆心的距离为 $E\left(d_{i}\right)-D\left(d_{i}\right) \approx 0.43 r$. 图 3 中的阴影部分表示的是圆格被距离最近的节点覆盖的部分, 它占到了整个 圆格的 73\%. 从图 2 中可以看出, 相邻的圆格有覆盖重叠的部分, 所以整个簇 内被工作节点所覆盖部分的比例要大于 $73 \%$,而且被工作节点覆盖的区域 较为均匀地分布在整个簇内,保证了网络可实时地、不间断地对移动目标进 行监测.

由于随机分布的节点可以被看作泊松点过程, 则一个簇内节点数目的 均值应当是 $\lambda\|A\|$, 即 $\frac{N}{\|S\|} \pi R^{2}$, 其中 $N$ 是节点部署总数, $S$ 为部署区域, $R$ 为簇

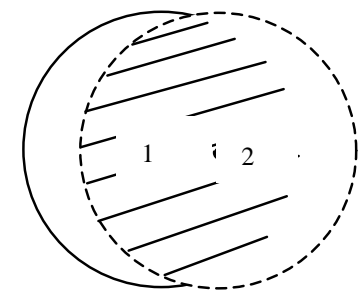

Fig.3 Coverage of nearest node

图 3 最近节点覆盖 半径.簇内调度算法是将簇成员遍历 1 遍,所以算法复杂度是 $O(N)$.

传感器监测网络大部分时间是处于空闲监测状态,为了进一步降低能耗,同时又要保证较好的监测质量,我 们在簇内再采用一种边界值守点调度的方法.当算法选定簇内的工作节点后, 让位于该簇边界的那些工作节点 一直处于活动状态, 其他工作节点进入睡眠状态并周期性地苏醒一段时间. 当边界节点发现移动目标后向簇首 报告,族首在簇内广播一个 wakeup 消息,收到该消息的工作节点不再睡眠而是转入监测工作状态.

\section{2 生成树的创建}

在生成树创建阶段,基站对网络进行广播.收到该广播的簇首记录下自己到基站的费用并广播该费用信息， 其他簇首在收到相邻簇首的费用信息后计算出自己到达基站的最小费用并转发出去,最终在全网范围内形成 一个簇首为节点的生成树.

定理 1. 在上述生成树创建过程中,每个簇首只广播 1 次最小费用消息即可建立最小费用生成树.

该定理可由文献[21]中的命题 3.1 直接推导出.

由于监测移动目标的网络对数据的实时性要求较高, 因此数据在通过生成树传回基站的过程中树上的各 个节点不对其进行汇聚而是直接向上游节点转发.

\section{4 仿真及性能分析}

\section{1 参数设置及性能指标}

在本文中我们使用 NS2 作为仿真平台,从 3 个方面衡量 EECTS 协议的性能.

簇首分布一一簇首的分布要均匀,因为簇首分布决定了本轮中网络的能耗.由式(1)可知,如果簇首分布得不 均匀,簇内的通信就不能满足式(1)中的自由空间模型,从而导致能耗成倍增加.

持续监测度 $\operatorname{CSD}$ (continuous surveillance degree)——假设移动目标经过整个监测区域的时间为 $T$, 仿真时 让传感器节点周期性地把对移动目标的监测结果传送回簇首, 汇报频率为 $k$ 次/秒. 因此, 我们将持续监测度定义 为移动目标经过整个监测区域时被发现的次数与 $(T \times k)$ 的比值. 比值越大对目标的持续监测能力越好. 为了防止 某条路径的特殊性而带来监测性能上的差异, 目标随机选择 3 条路径通过网络,下面仿真中的持续监测度的数 值都是 3 条路径上持续监测度的平均值.

网络寿命一一定义为网络对目标的持续监测度不小于 0.5 时所经历的时间.因为在这段时间内,网络可以对 移动目标进行有效的监测.

在仿真中使用的参数见表 1 . 
Table 1 Simulation parameters

表 1 仿真中使用的参数

\begin{tabular}{cccc}
\hline Parameter & Value & Parameter & Value \\
\hline Network size & $100 \times 100$ & Reprot frequency & $5 /$ second \\
Node number & $300,400,500,600$ & $E_{\text {elec }}$ & $50 \mathrm{~nJ} / \mathrm{bit}$ \\
Target speed & $10 \mathrm{~m} / \mathrm{s}$ & Round time $(T)$ & $10 \mathrm{~s}$ \\
Cluster radius $(R)$ & $20 \mathrm{~m}$ & Initial energy & $2 \mathrm{~J}$ \\
Sense radius $(r)$ & $5 \mathrm{~m}$ & Idle power $\left(P_{\text {idll }}\right)$ & $16 \mathrm{~mW}$ \\
Packet size $(Z)$ & 500 Byte & Bandwidth $(B)$ & $1 \mathrm{M} / \mathrm{bps}$ \\
$\varepsilon_{f s}$ & $13 \mathrm{pJ} / \mathrm{bit} / \mathrm{m}^{2}$ & $\varepsilon_{m p}$ & $0.0013 \mathrm{pJ} / \mathrm{bit} / \mathrm{m}^{4}$ \\
$\alpha$ & 0.5 & $\beta$ & 0.5 \\
\hline
\end{tabular}

下面仿真中的数据都是经过 20 次仿真后得到的平均值. 其中,EECTS 协议分为 EECTS-1 和 EECTS-2(使用 边界点值守的方法).

HEED 协议采用的是 TDMA 调度, 假设网络部署 $N$ 个节点, 分成 $L$ 个簇, 每个簇中的节点数为 $n_{i}$, 一个时隙长 为 $s t$, 数据包大小为 $Z$, 网络带宽为 $B$, 在一个时隙内成功发送数据包的概率为 $p_{\text {suc }}$. 由于目标出现的时刻 $x$ 在 $[0, s t]$ 内服从均匀分布,则

$$
p_{\text {suc }}=\int_{0}^{s t-\frac{Z}{B}} \frac{1}{s t} \mathrm{~d} x
$$

从式(7)中可以推导出时隙长 $s t=\frac{Z}{B\left(1-p_{\text {suc }}\right)}$. 为了使数据包发送的成功率不低于 0.9 , 要求时隙的长度至少为 $40 \mathrm{~ms}$, 下面仿真中 HEED 所使用的时隙就是该值. HEED 协议在一轮中的能耗绝大部分是在空闲状态时消耗的, 可以算出在一轮中当簇形成后 HEED 协议的空闲能耗为

$$
L \times P_{\text {idle }} \times T+\sum_{i=1}^{L} \frac{T}{n_{i} \times s t} \times n_{i} \times s t \times P_{\text {idle }}=2 T L P_{\text {idle }}
$$

其中, $P_{i d l e}$ 表示空闲功率,由于 $T$ 和 $P_{\text {idle }}$ 是固定不变的,HEED 的能耗只与簇的数目有关.

\section{2 仿真结果及分析}

图 4 和图 5 分别表示了部署了 300 个和 600 个节点的网络运行到第 20 轮时的簇首分布图.从图中可以看 出,任意相邻簇首间的距离均大于簇半径 $R$, 而且簇首分布均匀.

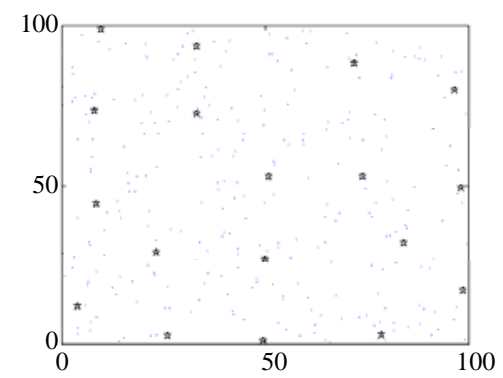

Fig.4 Distribution of clusterheads of 300 nodes

图 4300 个节点时簇首分布图

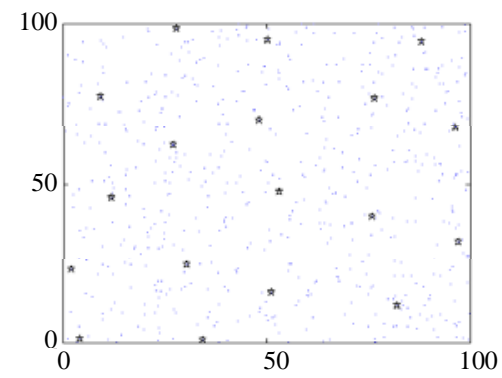

Fig.5 Distribution of clusterheads of 600 nodes

图 5600 个节点时簇首分布图

传感器监测网络最重要的任务就是能够对移动目标进行持续监测, 以获得目标的实时性数据. 图 6 是 EECTS 与 HEED 和 DEEG 两种协议对进入网络的移动目标的持续监测度的比较图.从图 6 可以看出, 在簇半径 均为 $20 \mathrm{~m}$ 的情况下,EECTS 对目标的持续监测度要远优于 HEED. 这是因为传统的分簇协议,如 HEED 等都是对 固定目标或现象进行监测, 采用的是 TDMA 调度, 在簇中的某个时刻只有一个节点处于活动状态, 当簇半径较大 时, 目标穿越一个簇被探测的概率是非常小的.EECTS-1 的簇内调度是根据图 2 的覆盖模型在簇内选择一部分 节点使其处于活动状态, 它保证了簇内的大部分区域可以被活动节点监测到;EECTS-2 是选择簇中边界节点值 
守,当移动目标进入网络后由簇首唤醒其他沉睡的节点,这两种方法都可以对移动目标进行持续监测. 随着节点 部署数目的增加,EECTS 协议在簇内选择的活动节点越来越接近覆盖模型中圆格的中心位置, 使得网络的覆盖 率趋于 1 , 对目标的持续监测度也最终趋向 1, 图 6 也反映了这一点. 从图 6 中我们注意到,DEEG 与 EECTS 的持 续监测度基本相同,这是由于 DEEG 协议从簇内选择了相当数量的活动节点,使得簇内的大部分区域可以被监 测到,所以,DEEG 对移动目标的持续监测度接近 EECTS.

图 7 是在网络部署 300 个节点时,HEED 协议的持续监测度与簇大小之间的关系.从图中可以看出,HEED 协议对移动目标的持续监测度随着簇半径的增加而迅速下降, 为了获得较高的监测度必须减小簇半径.下面在 网络寿命的仿真中,HEED 协议采用的是与 EECTS 取得相同持续监测度的簇半径.

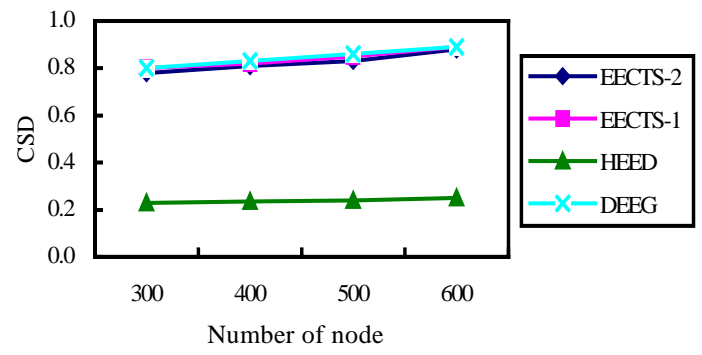

Fig.6 Continuous surveillance degree

图 6 持续监测度

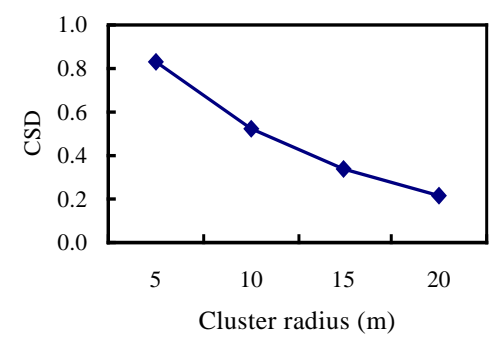

Fig.7 Continuous surveillance degree as cluster radius increases at HEED

图 7 持续监测度与簇半径(HEED)

图 8 和图 9 反映的是在持续监测度不小于 0.5 和 0.7 时的网络寿命.从图中可以看出,随着部署节点数目的 增加,EECTS,DEEG 和 HEED 三种协议的网络寿命都呈线性增长, 这是因为 EECTS 和 DEEG 两种协议在簇内 都是只选择了部分节点工作,其余节点处于休眠状态, 从而延长了网络寿命,HEED 协议采用节点轮转调度的方 式来节约能量. 由于 DEEG 是根据文献[11]中的式(11)确定工作节点的数目,并从簇成员中随机地选择,会使得节 点的监测区域重叠部分较多,而 EECTS 是根据图 2 中的覆盖模型来选择工作节点,因此在覆盖相同大小的区域 时,DEEG 要求的工作节点数目要多于 EECTS 的,直接导致了网络寿命的减小,从图 8 和图 9 中我们注意到,运行 EECTS-1 时的网络寿命比运行 DEEG 的网络寿命提高了约 35\%.图 5 表明,如果 HEED 协议想要取得和 EECTS 相同的持续监测度,其簇半径的大小必须约等于节点的探测半径, 这使得 HEED 协议与 EECTS-1 协议在一轮中 的活动节点的数目基本一样. 由式(8)可知,HEED 的能耗主要取决于簇的数目, 所以运行 EECTS-1 时的网络寿命 与运行 HEED 协议时网络的寿命基本一致,图 8 和图 9 中的结果也证明了这一点. 由于 EECTS-2 是在 EECTS- 1 选择的工作节点中选取了一部分边界节点,并让它们处于活动状态,进一步减少了能量的消耗,从图 8 和图 9 中 可以看出,运行 EECTS-2 的网络寿命比 EECTS-1 和 HEED 的高出约 70\% 80\%.

图 10 是部署 300 个节点时节点的存活数量与网络工作时间的关系图.从图中的实验结果可以看出,在运行 EECTS-2协议时,网络中第 1 个节点死亡到绝大部分节点死亡之间的时间间隔要远大于其他 3 种协议.EECTS- 1 与 HEED 协议由于每轮中活动节点的数目基本相同,所以节点的死亡时刻也比较接近. 为了取得相同的持续监 测度,DEEG 协议要比 EECTS 激活更多的活动节点, 所以节点的死亡时间要早于其他 3 种协议. 图 10 也从另一 个侧面验证了图 8 和图 9 中结果的正确性.

\section{5 结论及工作展望}

本文提出了一种分布式的能量有效的传感器监测网络成簇协议 EECTS. 在该协议中, 节点根据相邻节点的 分布情况及自己的剩余能量大小来竞选簇首. 由于监测网络的首要任务是对进入网络的移动目标进行不间断 的监测,所以 EECTS 采用了一种中心化的簇内调度方法 EECTS-1, 在簇内选择一部分工作节点, 这些节点可以 监测到网络的大部分区域, 并在此基础上提出了改进方法 EECTS-2.EECTS-2 仅让簇内选出的工作节点中位于 
簇边界的节点处于活动状态, 在确保监测质量的前提下节约了能量.为了进一步减少能量消耗,EECTS 将监测到 的数据通过多跳路由传回基站.实验结果表明,在持续监测度相同的情况下,EECTS-1 与 HEED 协议的网络寿命 基本一致,比 DEEG 高出约 35\%,EECTS-2 协议比 HEED 协议又提高了约 70\% 80\%,适用于移动目标监测等应 用.传感器监测网络往往部署在遥远或危险的区域,环境比较恶劣, 不满足自由空间模型, 通信信道不对称, 节点 故障率高, 在下一步的工作中, 我们要更贴近实际环境来设计协议, 将不规则的探测模型引入到工作中. 由于簇 首在监测应用中的关键作用, 为了防止其失效, 需考虑簇首的备份问题.

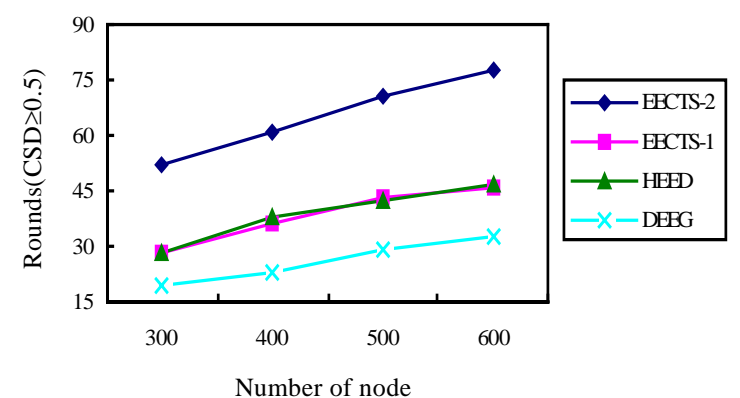

Fig.8 Network lifetime $(\mathrm{CSD} \geq 0.5)$

图 8 网络寿命(持续监测度 $\geq 0.5$ )

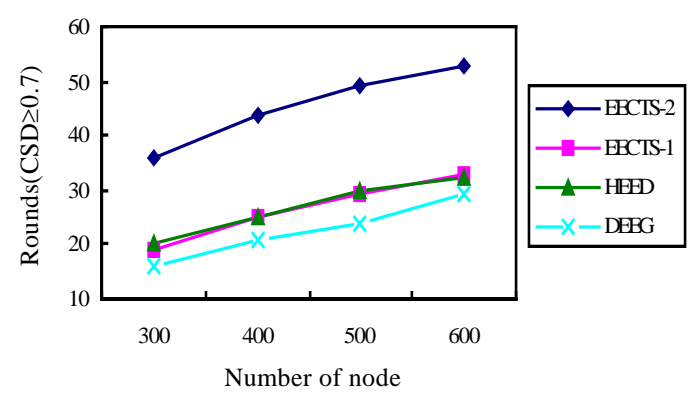

Fig.9 Network lifetime $(\mathrm{CSD} \geq 0.7)$

图 9 网络寿命(持续监测度 $\geq 0.7$ )
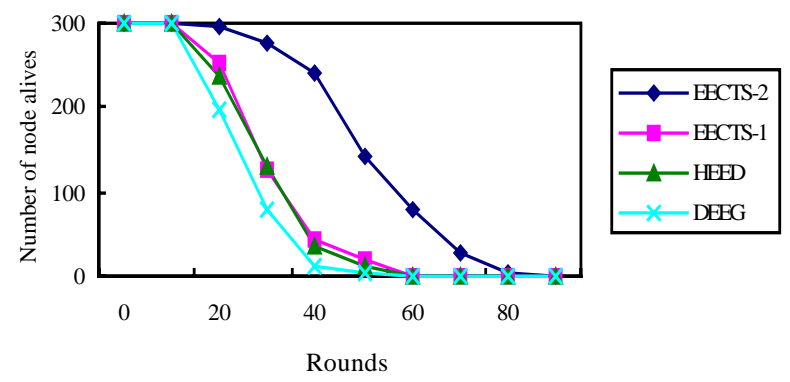

Fig.10 Time of node alives

图 10 节点存活时间图

致谢 在此,我们向对本文的工作给予支持和建议的导师,尤其是解放军理工大学指挥自动化学院齐望东教 授、中国电子设备系统工程公司戴浩院士及教研室的同学和老师表示感谢.

\section{References:}

[1] IF A, Weilian S, Yogesh S, Erdal C. Wireless sensor networks: A survey. Computer Networks, 2002,38(4):393-422.

[2] Deborah E, Ramesh G, John H, Satish K. Next century challenges: Scalable coordination in sensor networks. In: Kodesh H, Imielinski T, Steenstrup M, eds. Proc. of the 5th Annual ACM/IEEE Int'l Conf. on Mobile Computing and Networking (MobiCom). Seattle: ACM Press, 1999. 263-270.

[3] Kahn JM, Katza RH, Pister KSJ. Next century challenges: Mobile networking for "smart dust”. In: Kodesh H, Imielinski T, Steenstrup M, eds. Proc. of the 5th Annual ACM/IEEE Int'l Conf. on Mobile Computing and Networking (MobiCom). Seattle: ACM Press,1999. 271-278.

[4] Szewczyk R, Osterweil E, Polastre J, Hamilton M, Mainwaring A, Estrin D. Habitat monitoring with sensor networks. Communications of the ACM, 2004,47(6):34-40.

[5] Krishna P, Vaidya NH, Chatterjee M, Pradhan D. A cluster-based approach for routing in dynamic networks. Proc. of the ACM SIGCOMM’97, 1997,27(2):49-65.

[6] McDonald B, Znati T. Design and performance of a distributed dynamic clustering algorithm for ad-hoc networks. In: Jacobs A, ed. Proc. of the Annual Simulation Symp. New York: IEEE Press, 2001. 27-35. 
[7] Mhatre V, Rosenberg C, Kofman D, Mazumdar R, Shroff N. Design of surveillance sensor grids with a lifetime constraint. In: Karl H, Willig A,Wolisz A, eds. Proc. of the EWSN 2004. Berlin: Springer-Verlag, 2004. 263-275.

[8] Heinzelman WR, Chandrakasan AP, Balakrishnan H. Energy-Efficient communication protocol for wireless microsensor networks. In: Proc. of the Hawaii Int'l Conf. on System Sciences. San Francisco: IEEE Computer Society, 2000. 3005-3014.

[9] Heinzelman WR, Kulik J, Balakrishnan H. Adaptive protocols for information dissemination in wireless sensor networks. In: Kodesh H, Imielinski T, Steenstrup M, eds. Proc. of the 5th Annual ACM/IEEE Int'l Conf. on Mobile Computing and Networking (MobiCom). Seattle: ACM Press, 1999. 174-185.

[10] Younis O, Fahmy S. Distributed clustering in ad-hoc sensor networks: A hybrid, energy-efficient appoach. IEEE Trans. on Mobile Computing, 2004,3(4):660-669.

[11] Liu M, Gong HG, Mao YC, Chen LJ, Xie L. A distributed energy-efficient data gathering and aggregation protocol for wireless sensor networks. Journal of Software, 2005,16(12):2106-2116 (in Chinese with English abstract). http://www.jos.org.cn/ $1000-9825 / 16 / 2106$

[12] Ye M, Li CF, Chen GH, Wu J. EECS: An energy efficient clustering scheme in wireless sensor networks. In: Dahlberg T, Oliver R, Sen A, Xue GL, eds. Proc. of the IEEE Int'l Performance Computing and Communications Conf. New York: IEEE Press, 2005. $535-540$.

[13] Lindsey S, Raghavendra CS. Pegasis: Power-Efficient gathering in sensor information systems. In: Williamson DA, ed. Proc. of the IEEE Aerospace Conf., Vol. 3. New York: IEEE Press, 2002. 1125-1130.

[14] Tan HO. Power efficient data gathering and aggregation in wireless sensor networks. SIGMOD Record, 2003,32(4):66-71.

[15] Bandyopadhyay S, Coyle E. An energy-efficient hierarchical clustering algorithm for wireless sensor networks. In: Mitchell K, ed. Proc. of the INFOCOM 2003. New York: IEEE Press, 2003. 1713-1723.

[16] Hightower J, Boriello G. Location systems for ubiquitous computing. IEEE Computer, 2001,34(8):57-66.

[17] Elson J, Girod L, Estrin D. Fine-Grained network time synchronization using reference broadcasts. In: Proc. of the 5th Symp. on Operating Systems Design and Implementation. ACM Press, 2002. 147-163. http://lecs.cs.ucla.edu/Publications/papers/ broadcast-osdi.pdf

[18] Heinzelman WR. Application-Specific protocol architectures for wireless networks [Ph.D. Thesis]. Boston: Massachusetts Institute of Technology, 2000.

[19] Shih E, Cho S, Ickes N, Min R, Sinha A, Wang A, Chandrakasan A. Physical layer driven protocol and algorithm design for energy-efficient wireless sensor networks. In: Basagni S, Sivalingam K, eds. Proc. of the 7th Annual Int'l Conf. on Mobile Computing and Networking (MobiCom 2001). San Diego: ACM Press, 2001. 272-287.

[20] Benyuan L, Don T. A study of the coverage of large-scale sensor networks. In: Proc. of the ACM Mobile Ad-Hoc and Sensor Systems (MASS 2004). New York: ACM Press, 2004. 475-483. http://www.ececs.uc.edu/ cdmc/mass/mass2004/35142.pdf

[21] Ye F, Chen A, Lu S, Zhang L. A scalable solution to minimum cost forwarding in large scale sensor networks. In: Proc. of the 10th Int'l Conf. on Computer Communications and Networks. Arizona: IEEE Communications Society, $2001.304-309$. http://www.cs.ucla.edu/ alchemy/pubs/grab-icccn01.pdf

\section{附中文参考文献:}

[11] 刘明,龚海刚, 毛莺池, 陈力军,谢立.高效节能的传感器网络数据收集和聚合协议.软件学报,2005,16(12):2106-2116. http://www.jos.org.cn/1000-9825/16/2106

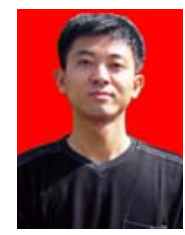

沈洋 $(1976-)$, 男,安徽蚌埠人,博士生, 讲 师,主要研究领域为指挥自动化理论与 技术.

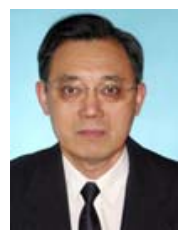

戴浩(1945-), 男, 研究员,博士生导师, 中 国工程院院士,CCF 高级会员, 主要研究 领域为指挥自动化理论与技术.

齐望东 $(1968-)$, 男,博士, 教授,博士生导师,CCF 高级会员, 主要研究领域为无线传感器网络. 